\title{
i-finepay: Platform Independent on the Spot Traffic Payment Solution
}

\author{
Navamalika P.D.P. \\ Faculty of Computing \\ Sri Lanka Institute of \\ Information Technology \\ Malabe, Sri Lanka
}

\author{
Jayawardena T.T. \\ Faculty of Computing \\ Sri Lanka Institute of \\ Information Technology \\ Malabe, Sri Lanka
}

\author{
Ranasinghe R.A.P.P. \\ Faculty of Computing \\ Sri Lanka Institute of \\ Information Technology \\ Malabe, Sri Lanka
}

\author{
Rupasinghe A.S.D. \\ Faculty of Computing \\ Sri Lanka Institute of \\ Information Technology \\ Malabe, Sri Lanka
}

\begin{abstract}
Society has been negatively influenced by the actions of the notorious drivers who proceeds to break the traffic rules. Active growth in the number of vehicle registrations in Sri Lanka is foreseen with the statistical evidence of vehicle registrations in 2018 being 7.7 million while it has increased by 305,303 in 2019 . With this increasing number of vehicles being registered and driven within the country, statistics showcase an increase in the number of traffic violations that occurred within the country. With the fast-moving lifestyles of the citizens, people tend to be wasting a significant portion of their time when intriguing to process through a traffic violation and its payment procedure. Not only the drivers but also the police officers face a hard time when tending to proceed with the validation and handling of documents related to violations including data analysis regarding the traffic violations. Through the proposed system, named i-finepay, it is designed to connect all the departments and personals that are entangled in this system of traffic violation and its payment, which includes the traffic police, the post office, the Sri Lanka Police department and the public. Through this system, it tends to ease the traffic violation and payment process for every user involved. This system also aids the Police officers in scouting and gathering information and preparing reports more efficiently. Moreover, some of the road violations will be detected through CCTV cameras and the fine will be figured out automatically in which the driver will be notified in real-time. Moreover, this system is embedded with image processing technologies to ensure high accuracy of data extraction and real-time processing for the validity of the data. With the processing of payments and the violations in real-time, the time of all the parties involved will be saved resulting in an efficient workload.
\end{abstract}

\section{Keywords}

Traffic violation, image processing, online payment, i-finepay

\section{INTRODUCTION}

Traffic violations are a common issue in every country, where notorious drivers have been violating traffic rules. This has been an increasing issue in Sri Lanka alongside the slow process of processing an accumulated violation. Sri Lanka has been practising the same paper-based methodology since the 1950 s with only some minor changes to the system. With this old methodology, the convicted party, as well as the police officers, tend to face numerous mishappenings while proceeding with the traffic violation management. The prevailing system, that is being practiced in Sri Lanka, takes place as follows.

Once a violation is done by a driver, the driver's license will be confiscated by the police officer and a fine sheet will be given to the drive. And then the driver will be asked to go back to the police station to get a fine payment sheet, which has to be paid from the post office and then once the payment is done, the driver is provided with a payment receipt which has to be returned to the police station of the police officer who confiscated his/her license, to get his/her license back.

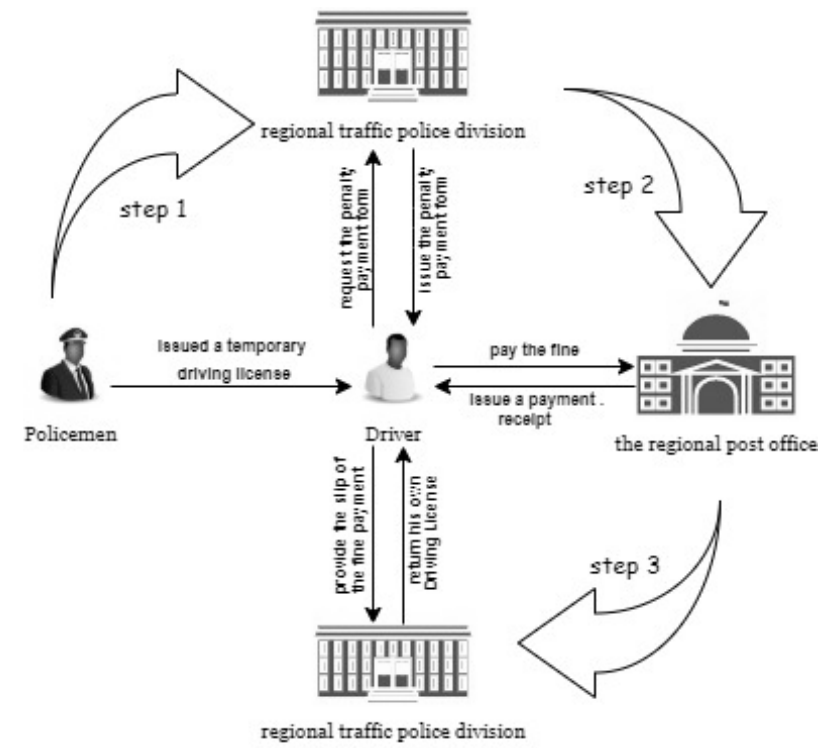

Fig 1: Process overview diagram of the existing system

With the prevailing system in Sri Lanka, a significant waste of time during a traffic violation. This scenario worsens if the driver has accumulated the violation on a Poya day as the post office will be closed on such public holidays. An example scenario of a worse case would be a driver who has been caught in a traffic violation on a Poya day who has been travelling out of their hometown. Once the driver is convicted of the violation, the driver's driving license will be confiscated, and the driver will be handed a fine sheet. However, as it is a public holiday the driver won't be able to make the payment on the same day and will have to travel back to get their license back. Not only the drivers but also the traffic police officers and the traffic police departments face a huge waste of time and resources throughout this process. The traffic police officer who happens to catch the driver in action for violating a traffic rule will have to trust his instincts in verifying the driver's license at first glance. If the traffic police officer feels it to be fake, then the officer will have to communicate with the department to validate the driver's license, which accumulates a significant amount of time for both parties involved. This same follows down towards the validation of the vehicle number plates of the convicted 
vehicle. Moving forward, the traffic department faces a huge workload and time consumption in analysing and finalizing the reports and statistical generation of any traffic violation analysis. Moreover, with the manual recording of the system data, the time taken for the processing of it increases and has less validity as some data can get lost or misread due to human errors that may occur. To avoid these obstacles flowchart for the proposed system is depicted by figure 2 . This flowchart was designed after a considerable analysis on the prevailing system and the obstacles faced by both parties.

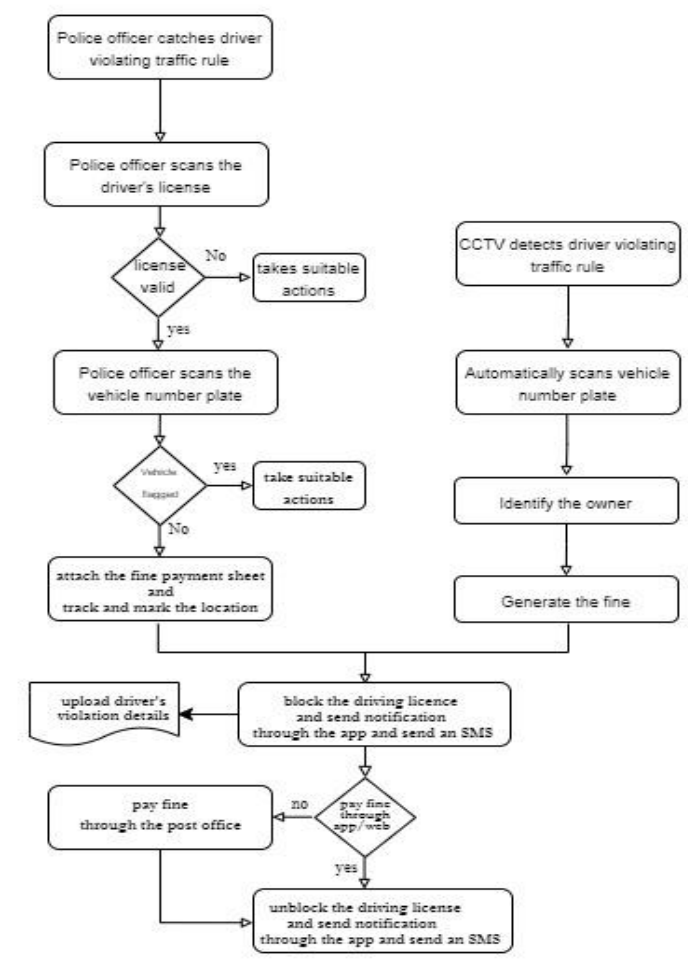

Fig 2: Flow chart of the proposed system

As a more efficient and effective solution for the prevailing system, a transition from the paper-based methodology to a more systematic computerized one is proposed. Here, the police officer will be presented with a mobile application. Through the mobile application, the police officer will be able to scan the driver's license to validate the driver's license and to view previous violations convicted by the driver. The system will be integrated with a status-based system, where the driver's license will have two statuses based on if the driver has been convicted for a violation. If the driver has accumulated a fine, the status of the license will be shown as blocked with a "blocked" status in the app, while if not it will be shown with a "valid" status. Next the officer can proceed with the validation of the vehicle number plate which will take a similar approach in the validation process as with the driver's license. Once completed these two steps, the police officer will be able to attach the written fine sheet to the system. The system will then automatically update the driver's license status and attach a fine to the driver's account while notifying the driver in real-time. Once the driver is convicted the driver will be able to pay the fine in two ways. One method is through the driver's app/ web application which will be integrated with an online payment gateway, or the driver will be able to pay through the post office which will be integrated with a system with online payment to instantly update the status of the driver's license and the details about the violations. Moreover, the proposed system is integrated with a module applicable for the traffic department which will be able to view and generate reports and analyse statistics through the system about different drivers and traffic violations. Also, this module will include a system integrated with CCTV cameras which will be actively monitoring for the speed violations of the vehicles. This module will automatically capture live feed from the camera and analyse to use it to determine speeding drivers who will be convicted with an automatic generation of the fine sheet through the system. The vehicle owners will be notified instantly about the violations.

The system enhances the accuracy of the data used in the system as it validates the data before being used in the system. Also, the online payment portal embedded in the driver's app/web application and the post office system ensures realtime data updating throughout the system. With the utilization of the CCTV cameras, the proposed system has been highlighted to provide easy and automatic usage of technology which were previously encountered through the police officers being physically available thus reducing the workload of the police officers. Also, with the usage of an integrated system, the traffic department can instantly generate and analyse data presented throughout the system with high accuracy and up to date information.

In this research paper, related works will be discussed in the literature survey. The next section it is discusses about the methodology of the system. Followingly it will ensure the testing and results of the proposed system and finally it will discuss about the further developments.

\section{LITERATURE REVIEW}

Traffic laws and regulations have been in place all over the world for over a century. Road users in most of countries tend to break traffic laws and safety standards thinking that the traffic police officers will not notice them. Increment in traffic violations creates a new problem for the traffic police officers in terms of enforcing educational consequences while maintaining deterrence. Traffic department do not possess enough workforce or resources to detect every inch of the road 24/7. Even so advancements were made in road safety regulations, implementation in middle- and low- income countries remain a key concern. According to the World Health Organization, traffic accidents cause more than a million lives worldwide each yearmostly due to illegal overtaking and exceeding speed limits, even some do not have the driving license with them, and traffic police officers will file cases for them with amercement and most of the drivers receive fines often knowing or without their knowledge. Automated traffic violation detection technology has been developing for decades. Some speed regarding issues have been addressed in developed countries using radars and backend systems but other violations like failure to wear the seat belt, failure to wear the helmet, failure to carry necessary documents are yet to be addressed. Most common sensors are CCTV cameras, which not only give a cost-effective solution but also provide extra features such as traffic surveillance and monitoring. Furthermore, smart systems designed using RFID, NFC technology, speed and line tracking sensors also have been proposed which is able to detect unauthorized speed and overtaking [1]. Moreover, SmartCop vehicle system to issue tickets, Traffic cameras, Speedometer clocks, LIDAR (light detection and range) needs a manual operator which is a major disadvantage, average speed computers, GPS, and GPRS systems that is to be attached to monitor and 
control the vehicle have been widely proposed and implemented regarding traffic violation issues. Major disadvantages of these systems are that it cannot work properly if the obstacles obstruct the signals as well, they are very much expensive considered to other alternatives.

Indian traffic police officers have begun to use various computerized traffic control systems in different states to encourage people to follow the road rules and regulations. Chirag Patel, Dipti Shah and Atul Patel introduced ANPR system which is an automatic number plate recognition system which gives different functionalities such as recognition and segmentation of characters, detection of number plates and image capture. As well, a system was proposed to install during the manufacture stage in order to detect the speed limits and notify the fine amount to the driver via SMS [2].Furthermore, some of these systems are able to capture violations and issue e-challans without any human participation as well some systems are able to generate echallans and send as an SMS to the violators with the evidence of a photo. In 2016, Traffic police of Delhi installed HD CCTV cameras in prominent locations, 50 cameras on traffic police cars, and 200 body-worn cameras. In 2017, another study which was conducted by Parul Shah, Sunil Karamchandani, Taskeen Nadkar, Nikita Gulechha, Kaushik Koli, Ketan Lad proposed an artificial neural network-based chassis number recognition algorithm based on OCR which was ideally suited for intelligent vehicular systems. An event driven traffic ticketing system was proposed with four modules event detection, recognizing plate number, database management and transmission of traffic ticket module to deliver tickets [3]. Though this system has different functionalities still there are some drawbacks regarding some factors like high speeds of vehicles, lightning conditions and different languages which will affect the overall recognition. Moreover, another system was proposed to change the traditional system to E-challan system where the traffic police officer can take a photo of the violator, which will be saved in their database, and the officer can create the violator's challan and finally the violation will be notified to the violator via SMS, and as well the payment options of the challan will be available for viewing [4]. Moreover, mobile traffic violation ticketing system was proposed in Egypt too. Belgium traffic police department is sending a letter to the person who has done the violation and he/she must insert the identification code and the police report number before proceeding to the payment. But this method is not fully automated due to the paperwork. Other than that, VANETS (Vehicular Ad Hoc Networks) have been proposed extensively by many researchers but there are many drawbacks such as the process is time consuming, hard to cover all insights of the road and hard to follow up multiple offenders [5].

Implementation of rules and regulations related to road traffic in Sri Lanka was established firstly under the Motor Traffic Act of 1951. Sri Lankan traffic police department do not use any automated system to handle the traffic related documents. Until today, Sri Lankan traffic police have followed the same laws and regulations, as well as the same fines for traffic offences, that have been implemented since the 1951 inception of the Motor Traffic Act. Since then, a few adjustments have been proposed that have had a minor impact on traffic rules and regulations. It is the case that any driver who is found breaking traffic laws must wait, wasting time on the roadways, until traffic officers physically write down each violation and other points on fine sheets. Furthermore, the traffic police officers demand to hand over the driver's license and to reclaim the license drivers must return to the post office to pay the fee amount [6]. A machine was proposed to install at police stations where people could pay for 33 fines through it making unnecessary to visit a post office to pay the fine. This system was mooted to overcome the obstacles such as the closure of post offices on weekends and public holidays. But this system was not yet implemented [7]. Another new system Motor Traffic Violation Monitoring System (MTVMS) was proposed where the users can register to the system as a voluntary service so that they can witness any violation and here they are able to upload live images and videos which would be needed in the verification process [8]. Furthermore, another web-based system was proposed which helps to connect the police department, traffic police officers, public and RMV officers. It enables police officers to add/ update fine details and generate reports. As well this system keeps the tracks of each record and an image of the driving license. With respect to the violation if a police officer produces a violator to the court system will also keep the court records [9]. Moreover, another system was introduced which consists of two parts as web-based information system traffic department and other authorities and the second part is analyzation of traffic violations using previous data. Another software was proposed for paying and maintain records regarding the traffic violations. This system has five types of users. They are main admin, traffic police officer in charge, traffic police offices, low enforcement authority and road users as well this consists of two parts. As the first step webbased information systems are introduced to traffic division and other related authorities. Secondly traffic violations are to be analyzed using previously collected data using rapid minor software to identify the violation pattern [10]. Even if it so, since these proposed systems are basic form filling systems can be some drawbacks such as typing errors happen during data entering to the system.

\section{METHODOLOGY}

The focus of achievement of this system is the traffic fine payment for violations accumulated by notorious drivers, and the management of those accumulated violations within a centralized network with the police department. The system overview diagram for the proposed system is depicted through figure 3. This system was designed with the data collection obtained through a survey conducted with a sample group of randomly selected personals from the public.

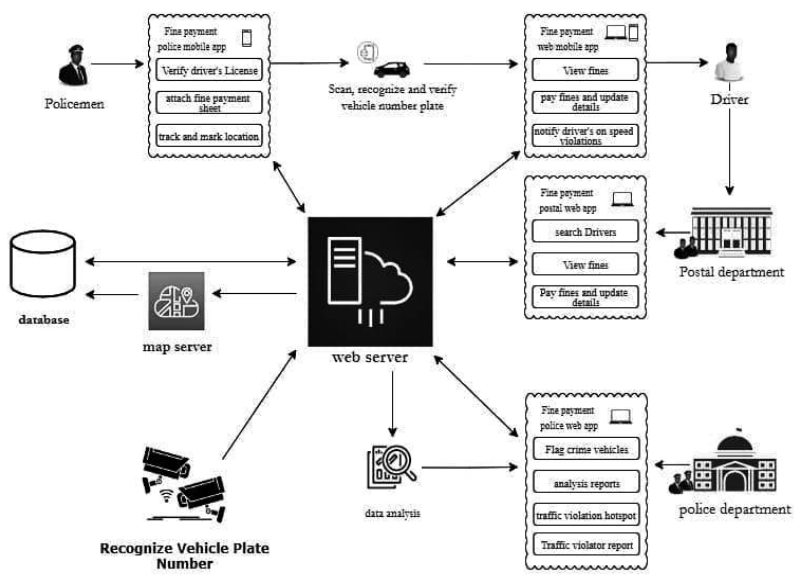

Fig 3: System overview diagram of the proposed system 


\subsection{Accumulating a violation}

Through the mobile application, the police officer will be able to validate the driver's license. The traffic police officer will be said to take a snapshot/photo of the driver's licensethrough the integrated camera module in the mobile app. This module will mainly focus on the close-range capturing of the driver's license to extract the driver's license number from the obtained image for verification. Once the image is captured successfully the image will be processed to obtain the driver's license number. The image will undergo a sequence of processing as to, it will be first turned into the grayscale mode to amplify the identification of dark areas/characters present in the photo. Once the image is greyscaled, the image will be undergoing the process of masking where the image is subjected to be further refined to shine out the specified locations of characters in the image. After successful masking of the image is completed, then the image will be passed through a pre-trained module of character recognition to identify every word present in the image captured. The captured words will be attached to a string that will concatenate a list of words that were extracted out of the $\mathrm{read} /$ processed image. This output string from the pre-trained module will then be passed through a function to filter and obtain the driver's license. Once the driver's license is obtained from the image, the driver's license number will be searched through the database to obtain its state and crossreferenced through the database again to get any accumulated violations. The system will be integrated with a status-based system, where the driver's license will have two statuses based on if the driver has been convicted for a violation at the time of validating. If the driver has accumulated a fine, the status of the license will be shown as blocked with a red background in the app, while if not it will be shown as good with a green background in the app.After the traffic police officer has reviewed the status of the driver's license and any accumulated violations if present, the traffic police officer can either proceed to check and verify the vehicle's number plate.

When the traffic police officer tends to proceed with the vehicle number plate verification, the traffic police officer will be prompted by a window where he is instructed to capture a photo of the vehicle's number plate. Once the number plate is captured automatic processing for the verification of the number plate will occur. Here the image of the vehicle number plate will be sent to be converted to a greyscale image and the masking of the image will take place. Once the masking of the image is completed, the image will be then sent through a pre-trained module of character recognition to identify the components/characters of the vehicle number plate. When the characters are successfully extracted a return string will be sent as output showcasing the number plate characters. These characters, as per the number plate, will be cross-referenced with the database to validate the vehicle's number plate and will check if the vehicle is flagged for any previously notified notorious deeds.

If the traffic police officer plans to proceed with fining the offensive driver, rather than just validating the driver's credentials, then the traffic police officer will be prompted with first verifying the license as described previously to validate the validity of the driver's license as well as to check for the driver's license status and any previous violation accumulations. Once the traffic police officer proceeds with the fining, then the validation screen for the vehicle's number plate is prompted. This procedure continues as described before and prompts if the vehicle is flagged and then proceeds to provide a summary of the full name, driver's license, and vehicle number plate. Once the police officer has completed writing the fine sheet, the police officer will be able to capture a copy/photo of the fine sheet and attach it to the system through the system. The system follows part of the old methodology of providing the fine sheet to the driver as part of the initiative as technology is not widespread within Sri Lanka and a significant part of the community is not equipped with a technology to view digital data. Thereby, as part of the initial stage, the system is developed to proceed with the fine sheet being written manually and handed over to the driver as proof of the use of the driver. Once the fine sheet is captured and submitted, the data in the fine sheet will be extracted before saving it to the database with the attached image. For the data extraction process of the fine sheet, once the fine sheet is submitted, it will be passed to a separate module, where the fine sheet will be processed for its data extraction. First and foremost, the fine sheet image will be converted into grayscale, and then the image will undergo a sequence of masking to enhance the image for better data extraction. After the masking is completed, the image will then be passed through a pre-trained module where the characters and words of the fine sheet will be read and extracted from the image. These extracted words will be attached to a string which will then be further processed to separate the features and be sent to be added to the database. For this process, the prevailing format of the fine sheet has been modified to suit betterhandwritten text recognition by minimizing the fields through which fewer characters are used, and more numerical data is passed such as rather than submitting the written name of the police station, it has been converted to provide the police station id instead. A sample copy of the redesigned fine sheet is attached below in figure 4 for reference. Once the data is successfully extracted from the fine sheet image, it will automatically be added to the database, and the relevant driver will receive an SMS message notifying about the fine that has been accumulated and the driver's license status will be updated as being blocked. Then the police officer will return the driver's license to the convicted driver.

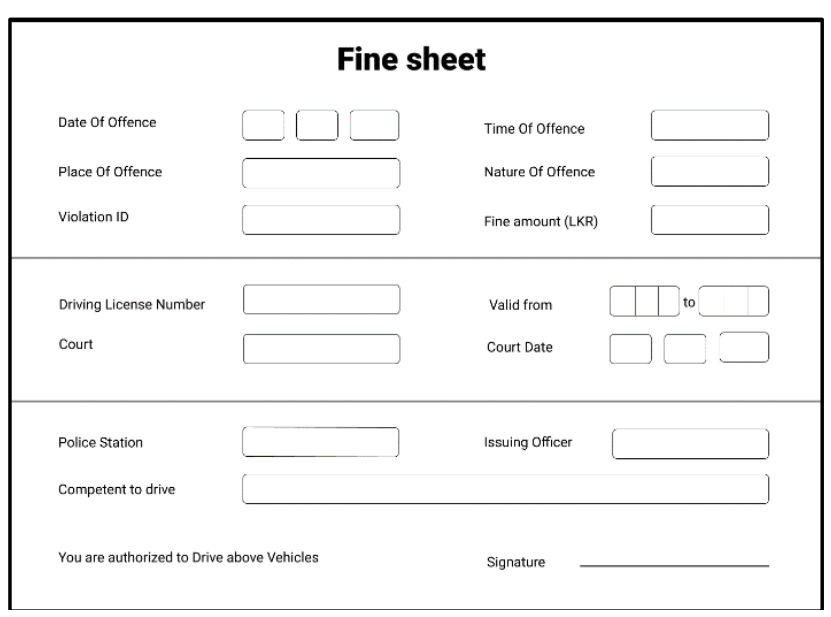

Fig 4: Re-designed fine sheet sample

\subsection{Paying the fine}

The driver who has accumulated a fine after the violation of a traffic rule will receive a message mentioning the fine once the traffic police officer has submitted the fine sheet. The driver can view their own driver's license status through the mobile application or the web application. Once logged into the system, the driver can view their current licensestatus as well as their accumulated violations if any. Once the driver refers to make the payment for the accumulated violation, 
they will be prompted with a payment window where they can fill in their card details and make an instant payment online. As soon as the payment is made, the system will be updated, and an SMS will be sent to the driver mentioning payment was made successfully and the driver's license status has been revered to being "valid."

In the case where the driver who has accumulated the violation has no means of paying via the online portal, then they are still capable of making the payment through the post office. The post office will be integrated with a web application through which the online payment module of the post office will be integrated. Through the post office portal, the post officer will be able to search for the relevant violations accumulated by a driver by entering the driver's driving license. Once the payment has been fulfilled, the post officer will be able to update the details of the payment through the portal. Once completed, the driver will receive an SMS mentioning that the driver has successfully made the payment and their license status is reverted to being "valid."

\subsection{Traffic monitoring and reporting}

All the data related to violations and fines that pass through the system can be monitored through a single location placed with the traffic department. This system is developed to manipulate and generate high-end reports about the violations occurring within the country by a central point of access. Once logged on to the system by the traffic department, they will be posted with an interface summarizing all the current data about violations and fines. One of the major components of this system is that the police officer will be able to flag a vehicle that has been accused/caught in action for a crime or a previous notorious activity. Through this, flagging off a vehicle, the officer will be capable of filling a form with the actual lawsuit, which then will be instantly updated such that the relevant data in the database will be instantly updated. By this, once a police officer scans the number plate of a vehicle, they will be able to proceed with the new and updated details instantly. Another major component of this module is the process of issuing a temporary license for a driver who has lost his license. Once the police officer has approved for the filing of a temporary license, the officer will be able to issue a temporary license by cross-checking the details through the system, using the driver's NIC. Once prompted with the result, the police officer will be able to instantly update the records and print a temporary license for the driver. Report generation and statistics is also an integral component in this module. Here the data regarding the violations and fines will be processed to produce a report for the analysis of the department. Through this module, the traffic department will be capable of identifying locations/areas of major traffic violations and access drivers who are being accused of frequent notorious driving.

A key component that will be integrated to the traffic department is the integration of automatic speed detection through cameras. Here, in this module, CCTV cameras will be used to automatically capture a speeding vehicle. The camera will be placed at a specific angle from a significant distance to the vehicle, such that the vehicles are captured clearly. Through the camera the system will calculate the speed of the vehicle from the live footage. Any vehicle that will exceed the speed limit will be captured and the image will be processed for reading the vehicle number plate. Once the image is captured the image will be cropped to ensure only the number plate is being processed. Once the cropped image is obtained, it will then be converted to greyscale and be processed to enhance the image through masking to ensure the image is properly suited for processing. After the masking process, the image will be passed through a pre-trained module which will then process the image and the license number will be returned. By obtaining the license plate number, the system will then cross-reference the number plate with the database to capture the owner of the vehicle, who will then be accumulated with a violation. An automatic fine will be generated for the owner of the vehicle and the owner's driver's license status will be "blocked." Even if the owner happens to not have a driver's license the owner will receive an SMS with the violation details.

\section{TESTING/EVALUATION AND DISCUSSION}

This chapter highlights, analyze the work and factors out the limitations of the prevailing system.Mainly this system aims to reduce paperwork while ensuring the confidentiality and security of the related documents since there is lot of paperwork involved in the prevailing fine system, avoid occurring delays during the process, maintain reliable payment methods, save resources as well the time and ease the work. Furthermore, any citizen of Sri Lanka can login to the system and check their fine payment history and the prevailing fines if any. Moreover, the police officers and the drivers can view their violations and fine amount which makes the case transparent, bribery will be impossible and cheating between the drivers and police officers when they get caught in a traffic violation will be reduced. As a result of using image processing technologies for scanning the driver's license and the vehicle number plates human errors and typing errors will be minimized throughout the process. Furthermore, driver's license status will be blocked, and license will be given back so that he/she does not need to travel back to the police station where the violation had taken place as well, SMS notification will be sent to the guilty party. As well in this system the violated vehicles can be flagged so that the relevant vehicle details will be saved in the online database. After the data regarding traffic violations has been saved in the database, reports and statistics about violation spots can be generated. Data will be analyzed on the web server, and once accessed, the traffic department web application will provide a graphical representation and take necessary steps to avoid them. Since there are people who still does not use new technology, capture a copy/photo of the fine sheet, and attach it to the system after police officer completed writing the fine sheet. Here, the data in the fine sheet will be extracted before saving it to the database with the attached image. Moreover, the impact of proposed system and the prevailing system was generated by utilizing the equations (1) and (2). Variables can be declared as $T_{p}=$ time spent at the officer, $T_{t s}=$ travel time to station, $T_{t p}=$ time spent at the post office, $T_{r}=$ time spent to release the license

Total time spent to retrieve license and pay fine $=$ $\sum\left(T_{p}+T_{t s}+T_{t p}+T_{r}\right)$

$L_{w h}=$ lost working hours, $T_{E p h}=$ GDP earned per hours, $T_{f p d}$ $=$ total fines per day, $T_{w d p y}=$ working days per year

Total annual GDP loss $=\prod\left(\left(\left(L_{w h} * T_{E p h}\right) * T_{f p d}\right) *\right.$ $T_{w d p y}$ ) 
Statistical analytics of the impact of the proposed system than the prevailing system is depicted by figure 5 and 6 while the table shows the testing summary for the whole system. Here, graph has been plotted considering the human errors, time, violation rates and manual labor of prevailing system with the proposed system. This depicts that a considerable percentage of defects have been reduced through the proposed system.

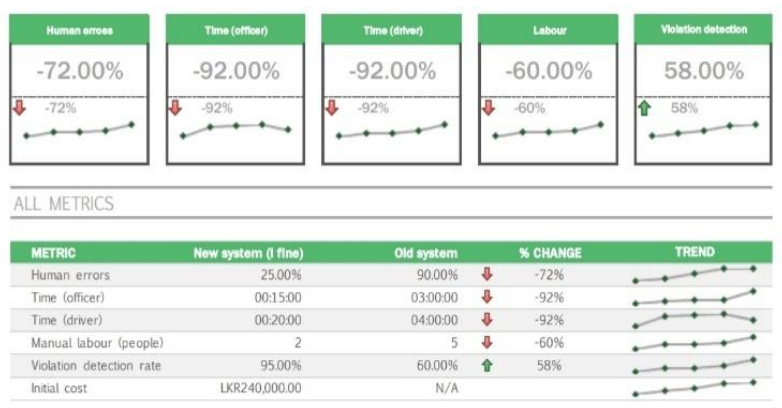

Fig 5:Impact of the proposed system

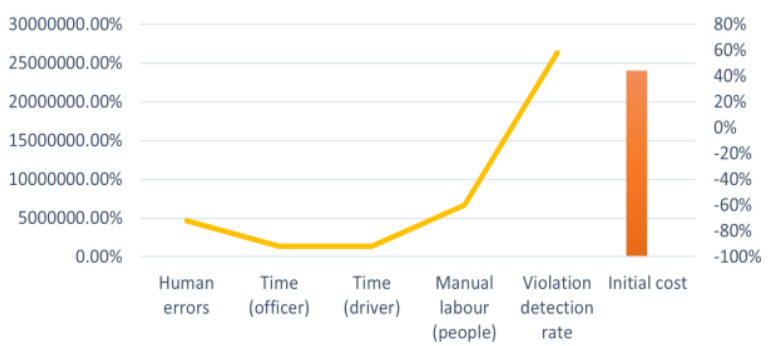

Fig 6: Estimated impact

Table 1. Testing Summary

\begin{tabular}{|c|c|c|c|}
\hline Scenario & Input & Output & Pass/Fail \\
\hline $\begin{array}{l}\text { Scanning } \\
\text { the driver's } \\
\text { license }\end{array}$ & $\begin{array}{l}\text { Image of the } \\
\text { driver's license }\end{array}$ & $\begin{array}{l}\text { Extracted license } \\
\text { number, license } \\
\text { status, violation }\end{array}$ & Pass \\
\hline $\begin{array}{l}\text { Scanning } \\
\text { the vehicle } \\
\text { number } \\
\text { plate }\end{array}$ & $\begin{array}{l}\text { Image of the } \\
\text { vehicle } \\
\text { number plate }\end{array}$ & $\begin{array}{l}\text { Extracted number } \\
\text { plate, vehicle } \\
\text { flagged status }\end{array}$ & Pass \\
\hline $\begin{array}{l}\text { Vehicle } \\
\text { flagging }\end{array}$ & $\begin{array}{l}\text { Details about } \\
\text { the flagged } \\
\text { vehicles }\end{array}$ & $\begin{array}{l}\text { Update the } \\
\text { vehicle status to } \\
\text { "flag" }\end{array}$ & Pass \\
\hline $\begin{array}{l}\text { Scanning } \\
\text { the fine } \\
\text { sheet }\end{array}$ & $\begin{array}{l}\text { Image of the } \\
\text { fine sheet }\end{array}$ & Fine sheet details & Pass \\
\hline $\begin{array}{l}\text { Attaching } \\
\text { the fine } \\
\text { sheet to the } \\
\text { driver's } \\
\text { account }\end{array}$ & $\begin{array}{l}\text { Image of the } \\
\text { fine sheet }\end{array}$ & $\begin{array}{l}\text { Fine sheet details, } \\
\text { update the license } \\
\text { status, sending an } \\
\text { SMS notification } \\
\text { about the fine }\end{array}$ & Pass \\
\hline $\begin{array}{l}\text { Paying the } \\
\text { fine sheet }\end{array}$ & $\begin{array}{l}\text { Payment } \\
\text { details }\end{array}$ & $\begin{array}{l}\text { Payment success } \\
\text { message, Sending } \\
\text { a SMS, updated }\end{array}$ & Pass \\
\hline
\end{tabular}

\begin{tabular}{|l|l|l|l|}
\hline & & license status & \\
\hline $\begin{array}{l}\text { Automatic } \\
\text { speed } \\
\text { detection }\end{array}$ & $\begin{array}{l}\text { Live CCTV } \\
\text { footage }\end{array}$ & $\begin{array}{l}\text { Detection of } \\
\text { speed violated } \\
\text { vehicles, sending } \\
\text { a SMS } \\
\text { notification }\end{array}$ & \\
& & Pass & \\
\hline
\end{tabular}

\section{CONCLUSION}

Once the 'i-finepay' application is hosted on a live environment, a strategic transformation in positive light can be expected in Sri Lankan traffic fine payment process. Since this is fully software based and smart devices-based system, this will be fruitful in changing existing procedures, to a system that is user friendly, secure and intelligent. Therefore, the system had to be designed in a user-friendly and very simple interfaces for better understanding. Slight changes were done to the database structure since there was no specific place to gather data. Another concern was the technology language. Since the technologies were unfamiliar a lot of time was spent in correcting the errors faced. This system prioritizes the speed, user-friendliness and reliability, and the reduction of paperwork. Furthermore, this is simple and secure. As for the future enhancements, this system which was originated from a concept and progressed up to this level should have a legal framework to make it more efficient and effective. Therefore, legal authorities and institutions like motor traffic department should make a proper implementation to make the best out of this system. In the future system can be fully digitalized removing all the paperwork by introducing the new technology for each citizen of Sri Lanka. Moreover, improving the drivers' license according to the system, will gain more effective results.

\section{REFERENCES}

[1] O. Nejati and M. Suraki, "NFC : Smart Recording Of Traffic Violation System", The 6th International Conference on Application of Information and Communication Technologies, 2012.

[2] P. R. Kambadkone, G. P. Hancke and T. D. Ramotsoela, "Real time speed detection and ticketing system," 2017 IEEE AFRICON, 2017, pp. 1593-1598, doi: 10.1109/AFRCON.2017.8095720.

[3] R. Mishra et al., "Analyzing Traffic Violations through e-challan System in Metropolitan Cities (Workshop Paper)," 2020 IEEE Sixth International Conference on Multimedia Big Data (BigMM), 2020, pp. 485-493, doi: 10.1109/BigMM50055.2020.00081.

[4] R. Srinath, J. Vrindavanam, Y. R. Sumukh, L. Yashaswini and S. S. Chegaraddi, "Smart Vehicle Recognition And E-Challan Generation System," 2020 International Conference for Emerging Technology (INCET), 2020, pp. 1-4, doi: 10.1109/INCET49848.2020.9154099.

[5] S. H. Ahmed, M. A. Yaqub, S. H. Bouk and D. Kim, "Towards contentcentric traffic ticketing in VANETs: An application perspective," 2015 Seventh International Conference on Ubiquitous and Future Networks, 2015, pp. 237-239, doi: 10.1109/ICUFN.2015.7182541.

[6] P. Madara, "Online Traffic Payment System", Research Gate,

2020 . 
Available:http://dx.doi.org/10.13140/RG.2.2.24387.2256 7.

[7] S. Ismail, "Online payment for traffic fines", Sunday Times, 2021.

[8] R. Wedage, "MOTOR TRAFFIC VIOLATION MONITORING SYSTEM FOR SRI LANKA POLICE", Dl.ucsc.cmb.ac.lk, 2021. [Online].Available: http://dl.ucsc.cmb.ac.lk/jspui/handle/123456789/4418.
[9] S. Wickramasinghe, "Implementation of online motor traffic violation management system", Dl.ucsc.cmb.ac.lk, 2021. [Online]. Available: http://dl.ucsc.cmb.ac.lk/jspui/handle/123456789/4516.

[10] B. TG, "Decision support system for road traffic violations", Dl.lib.uom.lk, 2021. [Online]. Available: http://dl.lib.uom.lk/handle/123/1594 